\title{
Interfacial Characteristics of Poly(methyl methacrylate) Film: Aggregation of Pyrene and Micropolarity Revealed by Time-Resolved Total Internal Reflection Fluorescence Spectroscopy
}

\author{
Akira Itaya, ${ }^{*}$ Takashi Yamada, Kenji Tokuda, and Hiroshi Masuhara* \\ Department of Polymer Science and Engineering, Kyoto Institute of \\ Technology, Matsugasaki, Sakyo-ku, Kyoto 606, Japan
}

(Received January 25, 1990)

\begin{abstract}
Fluorescence spectra and decay curves of pyrene in poly(methyl methacrylate) films, cast and spin-coated on sapphire substrates, were measured under a total internal reflection (TIR) and a normal excitation conditions and compared with each other. The difference in the intensity ratio of the first to the third vibronic bands of the pyrene monomer fluorescence was observed, which indicates that micropolarity around pyrene molecules in the vicinity of the sapphire/polymer interface is higher than that of the bulk. The intensity ratio of the excimer to the monomer fluorescence under the normal condition was larger than that under the TIR one. This result and the difference in the fluorescence decay curves under both optical conditions mean that an aggregate state of pyrene is different between the vicinity of the interface and the bulk. Similar phenomena were also observed for an air/polymer interface of the cast film. These interfacial characteristics of molecularly doped polymer films are discussed, and the formation process of these films is considered.
\end{abstract}

KEY WORDS Poly(methyl methacrylate) Film / Interface / Surface / Total Internal Reflection / Fluorescence / Pyrene / Aggregation / Micropolarity /

Surface and interface in polymer systems have received a great attention in recent science and technology. Conformation and orientation of polymers, and dopant distribution may change from the bulk to the interface, which results in characteristic physical and chemical properties of their solutions and solids. One of the powerful tools for these studies is electron spectroscopy such as ESCA, SIMS, RBS, etc., and the dry surface can be elucidated. ${ }^{1}$

An optical spectroscopy is also fruitful in the studies of solid/solid, solid/liquid, and liquid/vapor interfacial systems. Particularly, attenuated total internal reflection infrared spectroscopy and total internal reflection (TIR) Raman spectroscopy have been applied to various kinds of polymer systems. ${ }^{2,3}$ When light traveling in a material with higher refractive index $\left(n_{1}\right)$ is incident upon an interface at an angle greater than the critical angle, although the light undergoes total reflection, there is some penetration of the excitation light into the material with the lower refractive index $\left(n_{2}\right)$ in the form of an evanescent wave. This evanescent wave can be used to selectively excite or stimulate polymers which are located in the vicinity of the interface. The fluorescence spectroscopy under total internal reflection condition is probably the most sensitive one, ${ }^{4,5}$ and we improved its time-resolution up to $10 \mathrm{ps}^{6,7}$ Now timeresolved TIR fluorescence spectroscopy is an effective tool for elucidating molecular and electronic properties of polymer in the surface and interface.

Photophysical, photochemical, and photoconductive studies on polymer films have been carried out and provided very interesting and important results on amorphous organic solids. Molecularly doped polymer films are one of

\footnotetext{
* To whom all correspondences should be addressed.
} 
such systems and used for the studies of photosensitization, photostabilization, and so on. Moreover, such a system is used frequently for elucidating energy migration as well as transfer mechanism and charge transport mechanism, ${ }^{8-10}$ since an appropriate polymerdopant pair can be chosen and the concentration of light-absorbing dopant can be adjusted. Most of these studies have been made on the assumption that dopant molecules are homogeneously distributed in polymer films and that microenvironment around their molecules is the same for all molecules in polymer films. Here we challenge this assumption and deal with their related problems.

In the present work, the TIR fluorescence spectroscopy was applied to poly(methyl methacrylate) (PMMA) films doped with pyrene, which is the most representative system studied for a long time..$^{8,11}$ This molecule shows unique excited properties such as sensitivity of the fluorescence vibronic structure to the environment ${ }^{12}$ and has the longest lifetime as the singlet excited state. Furthermore, it is known that pyrene forms excimer in concentrated solution and that it forms dimer or/and aggregate in a rigid matrix at high concentration. ${ }^{11,13-15}$ The change of micropolarity around pyrene molecule and the its aggregation near the interface have been investigated.

\section{EXPERIMENTAL}

Pyrene (Aldrich) was zone-refined or chromatographed on silica gel using $n$-hexane for elution. Chromatographed pyrene was sublimed under vacuum. PMMA was purified by reprecipitations twice from benzene or tetrahydrofuran solution with methanol. Polystyrene (PSt) was the same as that used before. ${ }^{16}$ Solvents were purified by usual methods.

Sapphire was used as an internal reflection element $\left(n_{1}=1.81\right.$ at $\left.310 \mathrm{~nm}\right)$ and the same as that used before. ${ }^{6,7,17-19}$ Polymer films were cast on sapphire plates from chlorobenzene solution containing PMMA or PSt $(15 w t \%)$ and a known amount of pyrene at room temperature and dried in vacuo for several hours. The thickness of these these films was ca. $50 \mu \mathrm{m}$. The refractve index of PMMA and PSt films $\left(n_{2}\right)$ is 1.53 and 1.68 at $310 \mathrm{~nm}$, respectively, so that the critical angle $\left(\theta_{c}\right)$ given by $\sin \theta_{\mathrm{c}}=n_{2} / n_{1}$ was calculated to be $57.7^{\circ}$ and $68.2^{\circ}$, respectively.

Schematic diagram for the measurement is shown in Figure 1. Fluorescence spectra and decay curves were measured under both optical conditions and compared with each other. Fluorescence spectra were measured with a spectrofluorometer constructed using a stabilized $500 \mathrm{~W}$ Xe lamp (Wacom XD-501 S), monochromators (Ritsu MC-2L and MC$30 \mathrm{~N}$ ), and a PM-55 (S-20) photomultiplier. The diameter of an excitation light was reduced by an aperture and focused to the edge of the sapphire by quartz lens with $f=100 \mathrm{~mm}$. An incident angle $(\theta)$ was adjusted to $68.0^{\circ}$ and $76.3^{\circ}$ for PMMA and PSt systems, respectively, and the TIR phenomenon was confirmed by the naked eye. Decay curves were measured with a nanosecond time-correlated single photon counting system with two monochroma-

(1) TIR

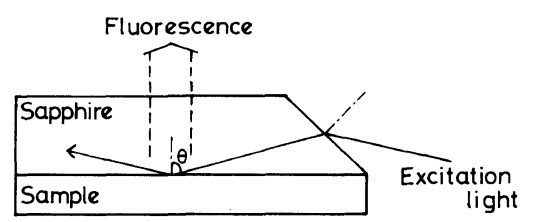

(2) Normal

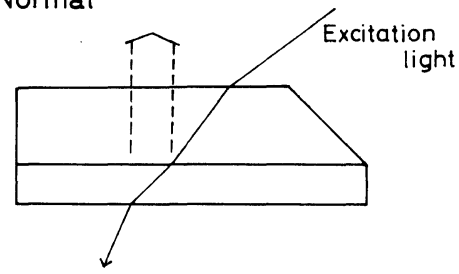

Figure 1. Schematic digram of fluorescence measurement. (1) Total internal reflection condition (TIR). $\theta$, incident angle. (2) Normal excitation condition (Normal). 
tors (Shimadzu Bosh-Lomb and Jobin-Yvon H-10) and a deuterium-filled flash lamp. The monochromatized excitation light pulse was used through a quartz lens and a pin-hole.

\section{RESULTS AND DISCUSSION}

\section{Micropolarity around Pyrene Molecules}

Fluorescence spectra of PMMA films doped with different concentration of pyrene are shown in Figure 2. These spectra were normalized at the third vibronic band $(0-2)$ of the monomer fluorescence. The film with low pyrene concentration $\left(7.29 \times 10^{-4} \mathrm{~mol} / \mathrm{MMA}\right.$ unit mol) shows a structured monomer fluorescence spectrum. For high concentration sample $\left(5.55 \times 10^{-2} \mathrm{~mol} / \mathrm{MMA}\right.$ unit mol$)$, a red-shifted broad excimer band was observed in addition to the monomer one. It has been reported that the excimer fluorescence increased with an increase in the pyrene concentration and that it did not arise from microcrystalline but from pairs of molecule with a parallel configuration. ${ }^{11}$ Here we notice
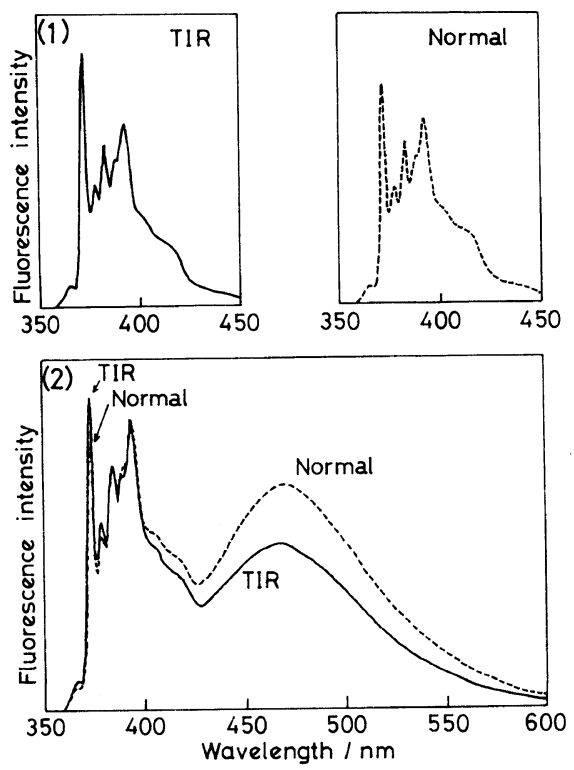

Figure 2. Fluorescence spectra of PMMA film doped with pyrene under the TIR and Normal conditions. Concentration: (1) $7.29 \times 10^{-4}$ and (2) $5.55 \times 10^{-2} \mathrm{~mol} /$ MMA unit mol. the following two points. One is that the fluorescence intensity ratio of the first (I) to the third (III) vibronic bands $\left(I_{\mathrm{I}} / I_{\mathrm{III}}\right)$ is larger under the TIR condition than under the normal one for both samples. Another is that the intensity ratio of the monomer to the excimer fluorescence under the TIR condition is larger than that under the normal one.

The difference of the vibrational structure of the monomer fluorescence is discussed here. Figure 3 shows a dependence of $I_{I} / I_{\text {III }}$ on the concentration of doped pyrene. If the low intensity of the first band under the normal condition is attributed to reabsorption, the difference between both conditions is expected to disappear in the low pyrene concentration. This is completely denied by the experimental result. Actually, an absorption coefficient of the $0-0$ absorption band of this dilute sample is very small $\left(2.6 \mathrm{~cm}^{-1}\right)$. The ratio of $I_{\mathrm{I}} / I_{\mathrm{III}}$ decreases with an increase in the concentration of pyrene for both conditions, which is attributed to a superposition of the excimer fluorescence and will be discussed later. It has been reported that the third vibronic band, which is strong and vibronically allowed, shows minimal intensity variation with polarity and the first band, which is forbidden vibronic one, shows a significant intensity enhancement in polar solvent. This is so-called Ham effect.

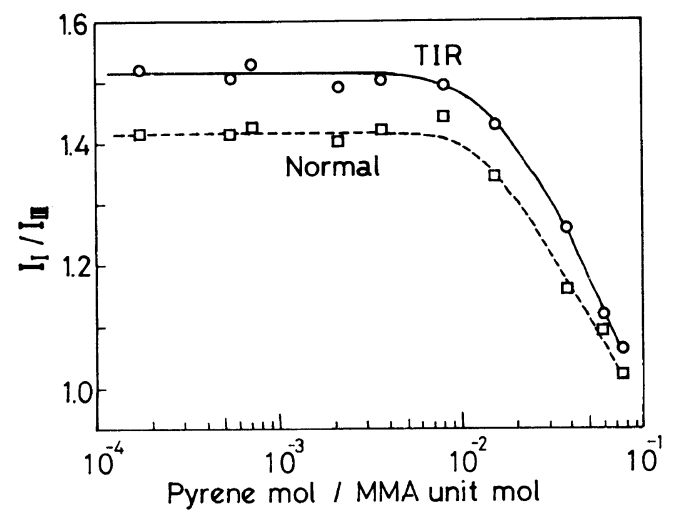

Figure 3. Dependence of the fluorescence intensity ratio of the first to the third vibronic bands $\left(I_{1} / I_{\mathrm{III}}\right)$ on the concentration of doped pyrene. 


\section{A. ITAYA et al.}
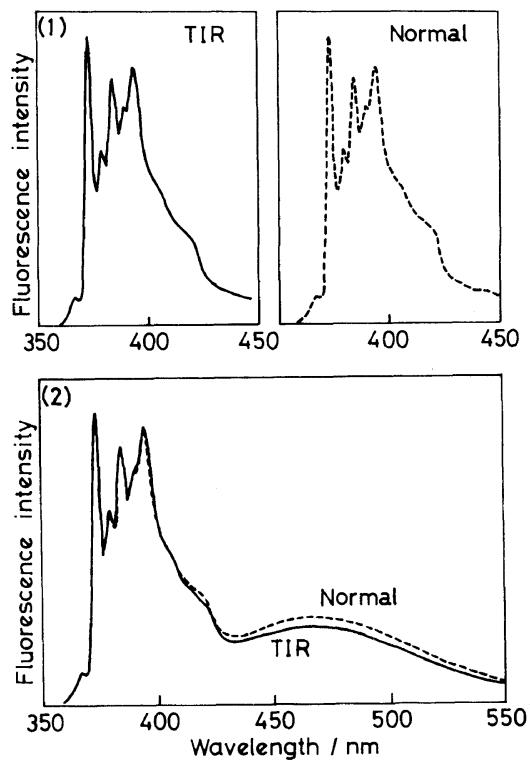

Figure 4. Fluorescence spectra of PSt films doped with pyrene under the TIR and Normal conditions. Concentration: (1) $2.08 \times 10^{-2}$ and (2) $5.06 \times 10^{-2} \mathrm{~mol} / \mathrm{St}$ unit mol.

Thus, the intensity ratio of $I_{\mathrm{I}} / I_{\mathrm{III}}$ of this molecule has been used as a probe for polarity of surrounding environments. ${ }^{12}$ According to this viewpoint, the present result means that micropolarity around pyrene molecules in the vicinity of the sapphire/polymer interface is higher than that of the bulk. We consider that the earbonyl group of PMMA is the origin of the micropolarity and that the degree of its orientation around pyrene is higher near the interface compared with in the bulk. In order to confirm this interpretation, PSt, which has no appreciable polar group, was selected as a matrix. Fluorescence spectra of PSt film doped with pyrene were examined under both optical conditions and are shown in Figure 4. The difference in the $I_{\mathrm{I}} / I_{\mathrm{III}}$ ratio is not observed between both optical conditions, of which the fact supports the interpretation just mentionedabove.

\section{Pyrene Aggregation}

As mentioned above, for the PMMA film doped with pyrene, the intensity ratio of the

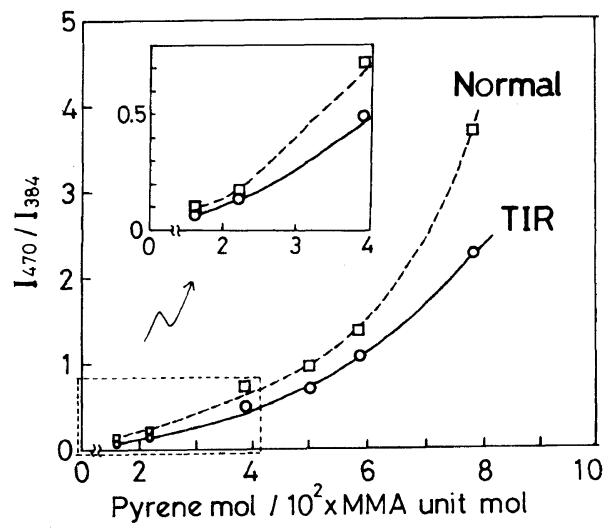

Figure 5. Dependence of the fluorescence intensity ratio of the excimer $(470 \mathrm{~nm})$ to the third vibronic band of monomer $(384 \mathrm{~nm})\left(I_{470} / I_{384}\right)$ on the concentration of doped pyrene.

excimer to the monomer fluorescence under the normal condition is larger than that under the TIR one. The same result was obtained for the PSt film doped with pyrene, as shown in Figure 4(b). Fluorescence spectra of PMMA films doped with various concentrations of pyrene were measured under both optical conditions, and intensity ratio of the excimer fluorescence peak $(470 \mathrm{~nm})$ to the third vibronic band of monomer one $(384 \mathrm{~nm})\left(I_{470} / I_{384}\right)$ is plotted against the concentration of pyrene in Figure 5. The third vibronic band was selected as a standard because of less sensitivity to the micropolarity. For a wide concentration range examined, the ratio of $I_{470} / I_{384}$ under the normal condition is larger than under the TIR one, and the ratio increases with an increase in the pyrene concentration. This suggests that the concentration of excimer forming pairs in the vicinity of the sapphire/polymer interface is lower than that in the bulk.

In order to confirm it, the monomer and excimer fluorescence decay curves were measured by monitoring at 374 and $520 \mathrm{~nm}$, respectively, under the both optical conditions. Typical decay curves are shown in Figure 6. Most of them obey non-exponential functions, so that the $1 / e$ lifetime of the fluorescence is used as a measure and plotted against the 


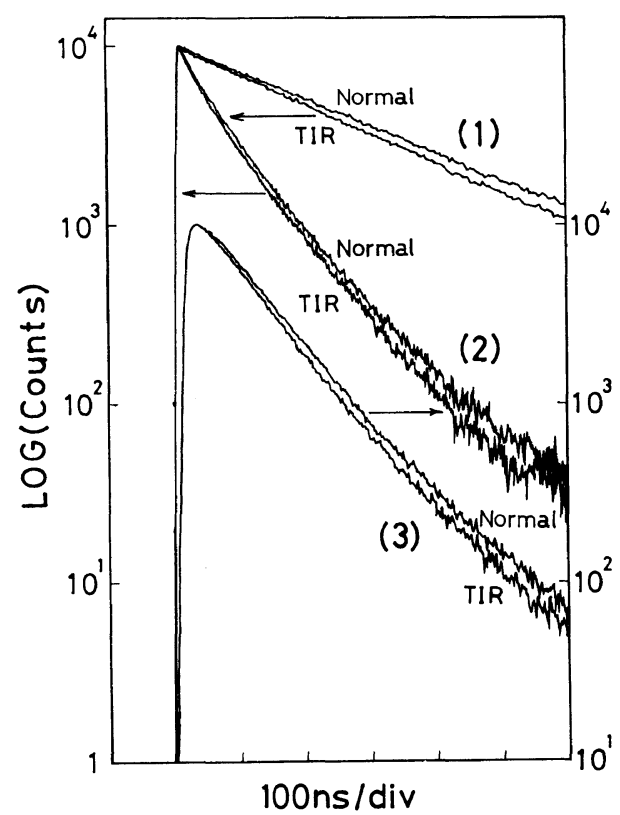

Figure 6. Fluorescence decay curves obtained by monitoring at $374 \mathrm{~nm}$ (Concentration: (1) $2.48 \times 10^{-3}$, (2) $5.58 \times 10^{-2} \mathrm{~mol} / \mathrm{MMA}$ unit mol), and (3) $520 \mathrm{~nm}$ (concentration, $4.90 \times 10^{-2} \mathrm{~mol} / \mathrm{MMA}$ unit $\mathrm{mol}$ ).

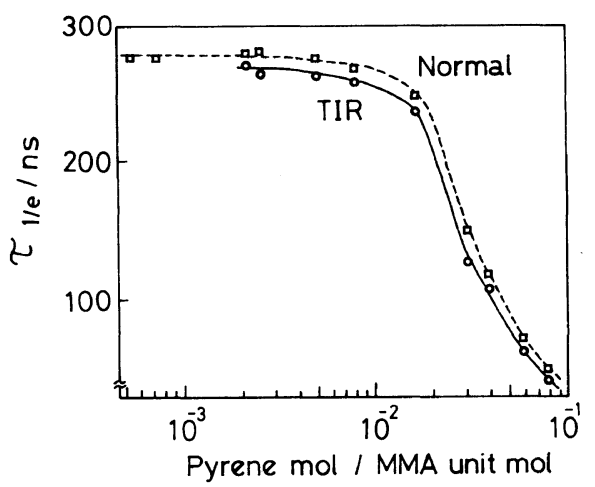

Figure 7. Dependence of the $1 / e$ lifetime of the monomer fluorescence on the concentration of doped pyrene. Decay curves of low concentration films under the TIR condition could not be detected because of low fluorescence intensity.

pyrene concentration in Figure 7 . The $1 / e$ lifetime under the TIR condition is smaller than that under the normal one for all concentration, of which the fact is in contrast to prediction from the above fluorescence result.

Many years ago Avis and Porter reported that an increase in the pyrene concentration in the same PMMA matrix leads to both an increase in the excimer fluorescence intensity and a decrease in a $1 / e$ lifetime of both the monomer and excimer non-exponential fluorescence decays and that excimer forming molecules (pairs whose geometry is close to the excimer configuration) and monomer molecules undergo relaxation processes almost independently. The decrease in the $1 / e$ lifetime of both fluorescence was considered to be attributed to a formation of dimer which is responsible for quenching of the fluorescence. ${ }^{11}$. According to this explanation, the present result indicates that excited pyrene molecules near the interface are more efficiently quenched by non-fluorescent pyrene dimer, compared to that in the bulk. That is, concentration of pyrene dimers leading quenching of the fluorescence is higher in the vicinity of the interface than in the bulk. Therefore, it is clear that, in the vicinity of the sapphire/polymer interface, the concentration of excimer forming dimer is lower and the concentration of the nonfluorescent dimer which is responsible for the quenching is higher as compared with the bulk.

\section{Thickness of Interface Layer}

We estimate here the depth from which the fluorescence we observed. Under the TIR optical condition, the effective thickness of the interface layer was calculated by applying the followings,

$$
\begin{gathered}
I=I_{0} \exp (-2 \gamma z) \\
\gamma=\left(2 \pi n_{1} / \lambda\right)\left(\sin ^{2} \theta-\left(n_{2} / n_{1}\right)^{2}\right)^{0.5}
\end{gathered}
$$

where $z$ is the depth from the interface, $I$ and $I_{0}$ are intensities of the excitation light at the interface and at the depth $z$, respectively, $\theta$ is an incident angle of the beam, $\lambda$ is its wavelength, and $n_{1}$ and $n_{2}$ are the refractive indices of the sapphire and polymer, respectively. Although these equations hold only for the light with $s$-polarization, qualitative estimations are possible. The intensity of the 
evanescent light becomes $1 / e$ of that of the interface at the depth of $0.036 \mu \mathrm{m}$ under the present experimental condition. The thickness under the normal condition depends upon absorbance, namely, the concentration of dopant and the excitation wavelength, and the depth where the excitation light intensity is $1 / e$ of the initial one is used as the effective thickness of the layer. At the excitation wavelength of $310 \mathrm{~nm}$, the thickness is $51.3,3.86$, and $0.476 \mu \mathrm{m}$ for low $\left(7.7 \times 10^{-4}\right)$, middle $(1 \times$ $\left.10^{-2}\right)$, and high $\left(8.1 \times 10^{-2} \mathrm{~mol} / \mathrm{MMA}\right.$ unit mol) concentrations of pyrene, respectively. We should point out that the fluorescence observed under the normal condition include the surface contribution which was obtained selectively under the TIR condition. At the present stage, we cannot argue whether the change of micropolarity and aggregation occurs continuously from the sapphire/polymer interface to the bulk or only at the interface. We can say safely that micropolarity and aggregation in PMMA films change in the depth region less than $0.476 \mu \mathrm{m}$ from the polymer/sapphire interface.

\section{Air/Polymer Interface}

We have also investigated the fluorescence behavior in the vicinity of an air/polymer interface. The polymer film doped with the concentration of $5.55 \times 10^{-2} \mathrm{~mol} / \mathrm{MMA}$ unit mol was stripped from the sapphire surface in deionized water, and the film was put on the sapphire with a contact between the sapphire and the air/polymer surface of the film with taking care to obtain a perfect optical condition. The difference in the intensity ratio $\left(I_{\mathrm{I}} / I_{\mathrm{III}}\right)$ of the vibrational band of the monomer fluorescence was hardly observed under both TIR and normal conditions. On the other hand, the intensity ratio of $I_{470} / I_{384}$ for the film under the normal condition is larger than that under the TIR one. The result on the $1 / e$ lifetime of the monomer fluorescence at $374 \mathrm{~nm}$ was also the same as that for the polymer/sapphire interface. Namely, pyrene aggregation near the air/polymer interface is the same as that for the polymer/sapphire one. We confirmed that these results were not induced by stripping in the deionized water. That is, the fluorescence behavior of the film, which was again put on the sapphire with the same contact between the sapphire and the polymer/sapphire interface as the original one, was measured and compared with that before stripping. No appreciable change was observed. The present result for the air/polymer interface indicates that the aggregate state of pyrene in the vicinity of the air/polymer interface is also different from that in the bulk.

\section{Spin-Coated Film}

We have also investigated spin-coated films doped with pyrene. The spin-coated films were prepared by using Mikasa spinner (1H-D2) and dried in vacuum for several hours. The film thickness was about $2.8 \mu \mathrm{m}$. All of the differences in fluorescence properties observed for the sapphire/polymer interface of cast films between TIR and normal optical conditions (the intensity ratio of the first to the third vibronic bands $\left(I_{\mathrm{I}} / I_{\mathrm{III}}\right)$ of the monomer fluorescence, the intensity ratio of the excimer fluorescence peak to the third vibronic band of the monomer one $\left(I_{470} / I_{384}\right)$, and the concentration dependence of the $1 / e$ lifetime at $374 \mathrm{~nm}$ ) were also confirmed for spin-coated films. It is concluded that the micropolarity around pyrene molecules and their aggregation near the polymer/sapphire interface in the case of spin-coated films is similar to that in the case of cast films. This suggests the formation process of film from solution is common for both films.

\section{Pyrene Excimer Structures}

As mentioned above, it should be noticed that the ratio of $I_{1} / I_{1 I I}$ decreases with an increase in the concentration of pyrene for both the TIR and the normal optical conditions (Figure 3). The similar behavior was also observed for PMMA films doped with various concentration 


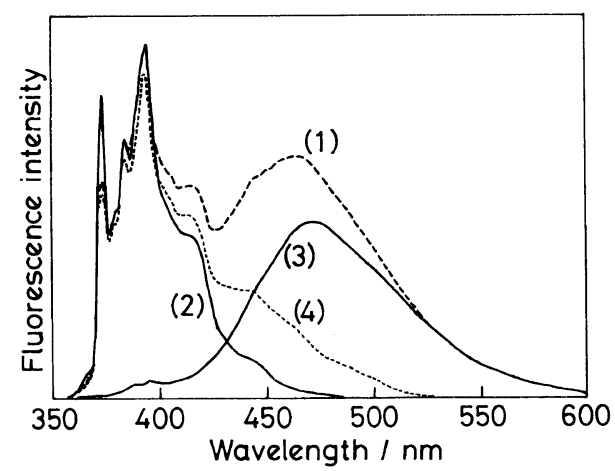

Figure 8. Fluorescence spectra of (1) (2) PMMA and (3) PSt films doped with pyrene. Concentration: (1) $3.9 \times 10^{-2}$, (2) $6.1 \times 10^{-4} \mathrm{~mol} / \mathrm{MMA}$ unit mol, and (3) $1.94 \times 10^{-1}$ $\mathrm{mol} / \mathrm{St}$ unit mol; (4) difference spectrum obtained by subtracting (3) from (1).

of pyrene (Figure 1 in ref 11) reported by Avis and Porter. However, it was not observed for PSt films (Figure 1 in ref 15) reported by Johnson. Fluorescence spectra of PMMA films doped with middle $\left(3.9 \times 10^{-2}\right)$, and low $\left(6.1 \times 10^{-4} \mathrm{mmol} / \mathrm{MMA}\right.$ unit $\left.\mathrm{mol}\right)$ concentrations of pyrene and PSt film doped with high $\left(1.94 \times 10^{-1} \mathrm{~mol} / \mathrm{styrene}\right.$ unit $\left.\mathrm{mol}\right)$ concentration of pyrene under the normal condition are shown in Figure 8. The spectrum of the highly doped PSt film was normalized at $530 \mathrm{~nm}$ to the fluorescence intensity of the middle one of PMMA films. The fluorescence intensity of the former film is very low in the wavelength region shorter than the third vibronic band of the monomer fluorescence. The difference spectrum which was obtained by subtracting the fluorescence spectrum of the highly doped PSt film from that of the middle one of PMMA film is not in agreement with the monomer fluorescence spectrum of the PMMA film doped with low concentration, and a weak broad fluorescence is superposed around $450 \mathrm{~nm}$ to the monomer one, as shown in Figure 8. The broad fluorescence is in the shorter wavelength region as compared with the normal excimer fluorescence, and hence it is assigned to partial overlap excimer. ${ }^{20}$ Therefore the decrease of the ratio of $I_{\mathrm{I}} / I_{\mathrm{III}}$ with an increase in pyrene concentration (Figure 3) is due to the superposition of the partial overlap excimer fluorescence on the third vibronic band of the monomer fluorescence. The result for PSt films reported by Johnson means that a formation of the partial overlap excimer is suppressed and the sandwich excimer is preferable in PSt films. Both excimers are involved in PMMA. This indicates that the aggregate state of pyrene molecules delicately depends upon properties of polymer matrix. Therefore, it is reasonable that the aggregate state of pyrene molecules in both polymer matrixes is different between the interface and the bulk.

\section{Interfacial Problem and Film Formation Proc- ess}

It is reported that there is a nonuniform concentration profile of polymer solution in the vicinity of a solid/liquid interface. The presence of the depletion layer of the polymer solution near nonadsorbing walls was confirmed experimentally by using an evanescent waveinduced fluorescence technique by Ausserre et $a .^{21-23}$ The depletion layer thickness decrease with increasing the concentration of the polymer solution. When the film was cast on the sapphire plate from the polymer solution as in the present work, it is considered that the depletion layer of the PMMA solution is formed in the vicinity of the sapphire/solution interface, and that its thickness decreases with an evaporation of the solvent. Local orientation of PMMA around pyrene in this region should be different from that of the bulk, and aggregation and its relative geometrical structure are also affected to a greater extent by this depletion layer.

On the other hand, the interfacial problem for the air/polymer interface is considered as follows. As soon as the sample solution containing PMMA and pyrene is dropped on the sapphire plate, evaporation of the solvent begins. The polymer concentration at the top of the solution layer reaches immediately a 


\section{A. Itaya et al.}

critical value for which the solution becomes gel, and a crust is formed. After then, the formation of the film proceeds under superposition of two phase, solid and fluid. The structure of the film (crust) formed by the sudden gel formation at the air/solution interface may be different from that by the slow evaporation of the solvent in the bulk. We consider that this difference in the mechanism of film formation is responsible for the difference in the aggregate state of pyrene between the air/polymer interface and the bulk. It is worth noting that micropolarity effect revealed by monomer fluorescence near the sapphire/polymer interface is appreciable, but not clear for the air/polymer interface. We consider that the orientation of carbonyl groups is related to the solid surface properties, while the dopant aggregation is determined by the polymer itself.

Acknowledgments. The present work was partly supported by the Grant-in-Aid for Scientific Research on Priority Area for Macromolecular Complexes (No. 63612510) and on Special Project Research for Photochemical Processes (No. 64104007), and the Grant-in-Aid for Scientific Research (No. 63430003) from the Ministry of Education, Science, and Culture of Japan.

\section{REFERENCES}

1. For example: D. M. Brewis, "Surface Analysis and Pretreatment of Plastics and Metals," Applied Science, London, 1982.

2. N. J. Harrick, "Internal Reflection Spectroscopy," Wiley-Interscience, New York, 1967.

3. R. Iwamoto, M. Miya, K. Ohta, and S. Miwa, J.
Chem. Phys., 74, 4780 (1981).

4. N. J. Harrick and G. I. Loed, "Modern Fluorescence Spectroscopy 1,” E. L. Wehry, Ed., Plenum Press, New York-London, 1976, p. 211.

5. B. K. Lok, Y-L. Cheng, and C. R. Robertson, J. Colloid Interface Sci., 91, 87 (1983).

6. H. Masuhara, N. Mataga, S. Tazuke, T. Murao, and I. Yamazaki, Chem. Phys. Lett., 100, 415 (1983).

7. H. Masuhara, S. Tazuke, N. Tamai, and I. Yamazaki, J. Phys. Chem., 90, 5830 (1986).

8. N. Mataga, H. Obashi, and T. Okada, J. Phys. Chem., 73, 370 (1969).

9. G. E. Johnson, Macromolecules, 13, 145 (1980).

10. J. Mort and G. Pfister, "Electronic Properties of Polymers," J. Mort and G. Pfister, Ed., WileyInterscience, New York, 1982, Chapter 6.

11. P. Avis and G. Porter, J. Chem. Soc., Faraday Trans. 2, 70, 1057 (1974).

12. K. Kalyanasundaram and J. K. Thomas, J. Am. Chem. Soc., 99, 2039 (1977).

13. J. B. Birks, "Photophysics of Aromatic Molecules," Wiley-Interscience, London, 1970, Chapter 7.

14. N. Mataga, Y. Torihashi, and Y. Ota, Chem. Phys. Lett., 1, 385 (1967).

15. G. E. Johnson, Macromolecules, 13, 839 (1980).

16. A. Itaya, K. Okamoto, and S. Kusabayashi, Polym. J., 17, 557 (1985).

17. Y. Taniguchi, M. Mitsuya, N. Tamai, I. Yamazaki, and H. Masuhara, J. Colloid Interface Sci., 104, 596 (1985).

18. A. Kurahashi, A. Itaya, H. Masuhara, M. Sato, T. Yamada, and C. Koto, Chem. Lett., 1413 (1986).

19. H. Masuhara, A. Itaya, A. Kurahashi, Y. Taniguchi, and M. Kiguchi, "Proceedings of the MRS International Meeting on Advanced Materials," Vol. 12, M. Doyama, S. Somiya, and R. P. Chang, Ed., Materials Research Society, Pittsburgh, 1989, p 321.

20. Y. Taniguchi, M. Mitsuya, N. Tamai, I. Yamazaki, and H. Masuhara, Chem. Phys. Lett., 132, 516 (1986).

21. C. Allain, D. Ausserre, and F. Rondelez, Phys. Rev. Lett., 49, 1694 (1982).

22. D. Ausserre, H. Hervet, and F. Rondelez, Macromolecules, 19, 85 (1986).

23. F. Rondelez, D. Ausserre, and H. Hervet, Ann. Rev. Phys. Chem., 38, 317 (1987). 